\title{
Some Epidemiological Aspects of Cutaneous Leishmaniasis in a New Focus, Central Iran
}

\author{
M. R. Yaghoobi-Ershadi, ${ }^{1}$ N. Marvi-Moghadam, ${ }^{1}$ R. Jafari, ${ }^{2}$ A. A. Akhavan, ${ }^{1}$ H. Solimani, ${ }^{3}$ \\ A. R. Zahrai-Ramazani, ${ }^{1}$ M. H. Arandian, ${ }^{2}$ and A. R. Dehghan-Dehnavi ${ }^{4}$ \\ ${ }^{1}$ Department of Medical Entomology and Vector Control, School of Public Health, Tehran University of Medical Sciences, Tehran, Iran \\ ${ }^{2}$ Isfahan Research Station, National Institute of Health Research, Isfahan, Iran \\ ${ }^{3}$ Yazd Health Research Station, National Institute of Health Research, Yazd, Iran \\ ${ }^{4}$ School of Public Health, Yazd University of Medical Sciences, Yazd, Iran
}

Correspondence should be addressed to N. Marvi-Moghadam; nr_moghadam@yahoo.com

Received 15 July 2015; Accepted 7 September 2015

Academic Editor: Iris Zalaudek

Copyright (C) 2015 M. R. Yaghoobi-Ershadi et al. This is an open access article distributed under the Creative Commons Attribution License, which permits unrestricted use, distribution, and reproduction in any medium, provided the original work is properly cited.

Following the epidemic of cutaneous leishmaniasis in Khatam County, Yazd Province, this study was carried out to determine vector, and animal reservoir host(s) and investigate the human infection during 2005-2006. Four rural districts where the disease had higher prevalence were selected. Sticky paper traps were used to collect sand flies during April to November, biweekly. Meanwhile rodents were captured using Sherman traps from August to November. Households and primary schools were visited and examined for human infection in February 2006. The parasite was detected by RAPD-PCR method. The rate of ulcers and scars among the inhabitants was $4.8 \%$ and $9.8 \%$, respectively. Three rodent species were captured during the study: Meriones libycus, Rhombomys opimus, and Tatera indica. Six sand fly species were also collected and identified; among them Phlebotomus papatasi had the highest frequency. Leishmania major was detected as the agent of the disease in the area. It was detected from R. opimus and native people.

\section{Introduction}

In the old world, cutaneous leishmaniasis $(\mathrm{CL})$ is a major public health problem in the Eastern Mediterranean Region of World Health Organization (WHO), with more than reported cases [1]. In this region, Iran is known as one of the high risk countries for the disease with more than 20000 annual cases. The endemic foci of CL are reported in 17 out of 31 provinces of the country while foci of Isfahan, Yazd, Khorasan-e-Razavi, Golestan, and Fars Province are more important [2-11].

Previous studies in Iran indicated Phlebotomus papatasi as the main vector among 45 reported sand fly species $[12,13]$. Rhombomys opimus and Meriones libycus are the main and secondary reservoir hosts and Leishmania major is the agent of zoonotic cutaneous leishmaniasis (ZCL) [2-11].

In recent years, CL cases have been reported increasingly from different areas of Yazd Province, central Iran, including Khatam County. Due to lake of data in this area, current study was designed and conducted to determine some epidemiological aspects of the statistical evaluation of patients with cutaneous in Health Center of Yazd during 2000-2004 that shows that most of cases exist in Khatam city, the southernmost province of Yazd There were 1509 cases recorded during these years. Most cases are in Herat (Fathabad Village) and Marvast (village of Harabarjan), and it has become a common disease in the last four years.

\section{Objects and Methods}

2.1. Study Area. Khatam County with $7931 \mathrm{~km}^{2}$ area and total population of about 35000 is located in the southeast of Yazd Province, central Iran. The elevation of the county ranges between 1500 and $3005 \mathrm{~m}$ above the sea level. The climate is hot and dry. Khatam County has two towns of Marvast and Harat as well as four rural districts of Fathabad, Harabarjan, Chahak, and Isar. Agriculture, animal husbandry, service affairs, office affairs, and trading are the main occupations 
Table 1: Prevalence of acute lesion and scar in the studied families, Khatam County, Yazd Province, central Iran, 2006.

\begin{tabular}{|c|c|c|c|c|c|c|c|c|c|c|c|c|c|c|c|}
\hline \multirow{3}{*}{ Age } & \multicolumn{5}{|c|}{ Males } & \multicolumn{5}{|c|}{ Females } & \multicolumn{5}{|c|}{ Total } \\
\hline & \multirow{2}{*}{ Visited } & \multicolumn{2}{|c|}{ Scar } & \multicolumn{2}{|c|}{ Acute lesion } & \multirow{2}{*}{ Visited } & \multicolumn{2}{|c|}{ Scar } & \multicolumn{2}{|c|}{ Acute lesion } & \multirow{2}{*}{ Visited } & \multicolumn{2}{|c|}{ Scar } & \multicolumn{2}{|c|}{ Acute lesion } \\
\hline & & Number & $\%$ & Number & $\%$ & & Number & $\%$ & Number & $\%$ & & Number & $\%$ & Number & $\%$ \\
\hline $0-4$ & 25 & 1 & 4 & 1 & 4 & 28 & 0 & 0 & 1 & 3.57 & 53 & 1 & 1.88 & 2 & 3.7 \\
\hline $5-9$ & 45 & 1 & 2.22 & 2 & 4.44 & 40 & 2 & 5 & 4 & 10 & 85 & 3 & 3.52 & 6 & 7.05 \\
\hline $10-14$ & 72 & 6 & 8.33 & 8 & 11.11 & 76 & 10 & 1.31 & 4 & 5.26 & 148 & 16 & 10.81 & 12 & 8.10 \\
\hline 15-19 & 124 & 16 & 12.9 & 5 & 4.03 & 134 & 6 & 4.47 & 6 & 4.47 & 259 & 22 & 8.49 & 11 & 4.24 \\
\hline $20-24$ & 98 & 6 & 6.12 & 1 & 1.02 & 76 & 7 & 9.21 & 2 & 2.63 & 174 & 13 & 7.47 & 3 & 1.72 \\
\hline+25 & 332 & 41 & 17.67 & 16 & 4.81 & 314 & 37 & 11.78 & 15 & 4.77 & 645 & 78 & 12.09 & 30 & 4.65 \\
\hline Total & 696 & 71 & 10.20 & 33 & 4.74 & 668 & 62 & 9.28 & 32 & 4.79 & 1364 & 133 & 9.75 & 65 & 4.76 \\
\hline
\end{tabular}

in the county (Yazd Province statistics 2005). The study was carried out in Fathabad, Harabarjan, and Marvast areas.

2.2. Study of Human Infection. Prevalence of cutaneous leishmaniasis was studied during February and 300 households were visited and interviewed. Family size, infected members, age, gender, disease condition (acute wound or scar), place or number of wounds, and time and place of infection were considered in the questionnaires. Smears were prepared and examined from the acute lesions of native people who had no history of travel to other endemic foci of the disease during past year. All students of the primary schools were also visited. The serosity of lesions was inoculated to the tail base of Balb/C mice in the field. After infection of the mice and wound formation, the serosity of their wounds was cultivated in the Schneider-RPMI media. After one week, promastigote stage of the parasite was developed and used for species identification using RAPD-PCR [14].

2.3. Study of Rodents. For this purpose wild rodents were captured around the study area during September to November biweekly using Sherman traps. Traps were installed before sunset and collected next early morning. Trapped rodents were identified using morphological and morphometric characteristics [15] and after anaesthesia two slides were prepared from each ear of the rodents [16]. Species recognition results and direct examination of parasitology were recorded by number, date, and trapping location.

2.4. Study of Sand Flies. Sand flies were collected biweekly from indoor (bedrooms, toilets, hall, etc.) and outdoor (rodent burrows) resting places using 30 sticky traps (castor oil coated on $\mathrm{A}_{5}$ white papers) from the beginning to the end of the active season. The traps were placed before sunset and collected next early morning. For species identification, sand flies were mounted in Puri's medium and identified using the relevant keys [17]. Sex ratio was calculated as the number of males/females $\times 100$. Study on sand fly infection was performed during August-September using dissection method and direct observation of head and gut under the light microscope. Susceptibility of sand flies to Deltamethrin was evaluated using WHO test kits in the diagnostic dose [1].
2.5. Parasite Detection. Schneider medium was used to transfer and culture the parasite in the field. The parasites then were transferred to RPMI medium in the laboratory and, after sufficient amplification, the species was identified using RAPD-PCR method [14].

2.6. Statistical Analysis. SPSS version 11.5 and $\chi^{2}$ test were used to analyse data.

\section{Results}

3.1. Human Infection. During February 2006 a total of 300 families with a population of 1364 (696 males and 668 females) were visited and their demographic as well as the disease data was recorded. Out of them, $4.64 \%$ had acute lesions (Table 1). $\chi^{2}$ analysis showed no significant difference between males and females $(\alpha=5 \%)$. Most of the wounds were found in 10-14 years age group (8.1\%), while the lowest rate was found in $20-24$ years age group $(1.72 \%)$. All students of primary schools of the area were also visited. The active lesions were found in $4.94 \%$ of them (Table 2 ). There was no significant difference between boys and girls $(P>0.05)$.

The isolated parasite from the local patients with no history of travel was detected as L. major.

3.2. Rodents. During this study 15 rodents were trapped and identified as follows: Meriones libycus (66.7\%), Rhombomys opimus (20\%), and Tatera indica (13.3\%). One M. libycus, one $T$. indica, and all $R$. opimus specimens were found to be infected with the amastigotes of Leishmania parasite (Figure 1). The isolated parasites were identified as L. major.

3.3. Sand Flies. A total of 5571 sand flies (772 indoors and 4999 outdoors) were collected and identified as Phlebotomus papatasi (85.35\%), Ph. mongoliensis (0.03\%), Ph. kazeruni (0.03\%), Ph. sergenti (0.03\%), Sergentomyia baghdadis (0.3\%), and Se. sintoni $(14.28 \%)$. The dominant species were found to be $P h$. papatasi and Se. sintoni, respectively. Phlebotomus mongoliensis and Ph. sergenti were collected only indoors, while Se. baghdadis was found only outdoors (Table 3). 
TABLE 2: Prevalence of acute lesion and scar in primary schools of the studied area, Khatam County, Yazd Province, central Iran, 2006.

\begin{tabular}{|c|c|c|c|c|c|c|c|c|c|c|c|c|c|c|c|}
\hline \multirow{3}{*}{ Age } & \multicolumn{5}{|c|}{ Boys } & \multicolumn{5}{|c|}{ Girls } & \multicolumn{5}{|c|}{ Total } \\
\hline & \multirow{2}{*}{ Visited } & \multicolumn{2}{|c|}{ Scar } & \multicolumn{2}{|c|}{ Acute lesion } & \multirow{2}{*}{ Visited } & \multicolumn{2}{|c|}{ Scar } & \multicolumn{2}{|c|}{ Lesion } & \multirow{2}{*}{ Visited } & \multicolumn{2}{|c|}{ Scar } & \multicolumn{2}{|c|}{ Acute lesion } \\
\hline & & Number & $\%$ & Number & $\%$ & & Number & $\%$ & Number & $\%$ & & Number & $\%$ & Number & $\%$ \\
\hline 7 & 77 & 3 & 3.89 & 2 & 2.59 & 71 & 4 & 5.63 & 4 & 5.63 & 148 & 7 & 9.52 & 6 & 8.22 \\
\hline 8 & 72 & 3 & 4.16 & 2 & 2.77 & 75 & 5 & 6.66 & 2 & 2.66 & 147 & 8 & 10.82 & 4 & 5.43 \\
\hline 9 & 59 & 4 & 6.77 & 2 & 3.38 & 52 & 3 & 5.76 & 1 & 1.92 & 111 & 7 & 12.53 & 3 & 5.3 \\
\hline 10 & 82 & 16 & 19.51 & 6 & 7.31 & 75 & 6 & 8 & 6 & 8 & 157 & 22 & 27.51 & 12 & 15.31 \\
\hline 11 & 70 & 17 & 10 & 5 & 7.14 & 63 & 7 & 11.11 & 4 & 6.34 & 134 & 14 & 21.11 & 9 & 13.48 \\
\hline 12 & 18 & 2 & 11.11 & 1 & 5.55 & 12 & 1 & 0.82 & 0 & 0 & 30 & 13 & 11.93 & 1 & 5.55 \\
\hline Total & 379 & 36 & 9.94 & 18 & 4.74 & 348 & 26 & 7.47 & 17 & 4.88 & 727 & 62 & 8.29 & 36 & 4.95 \\
\hline
\end{tabular}

TABle 3: Fauna, density, and sex ratio of sand flies in Khatam County, Yazd Province, 2006.

\begin{tabular}{|c|c|c|c|c|c|c|c|c|}
\hline \multirow{2}{*}{ Species } & \multicolumn{2}{|c|}{ Indoors } & \multicolumn{2}{|c|}{ Outdoors } & \multicolumn{2}{|c|}{ Total } & \multicolumn{2}{|c|}{ Sex ratio } \\
\hline & Number & $\%$ & Number & $\%$ & Number & $\%$ & Indoors & Outdoors \\
\hline Ph. papatasi & 735 & 95.08 & 4190 & 83.82 & 4925 & 85.33 & 383.5 & 159.76 \\
\hline Ph. mongoliensis & 2 & 0.26 & 0 & 0 & 2 & 0.03 & - & - \\
\hline Ph. kazeruni & 1 & 0.13 & 1 & 0.02 & 2 & 0.03 & - & - \\
\hline Ph. sergenti & 2 & 0.26 & 0 & 0 & 2 & 0.03 & - & - \\
\hline Se. baghdadis & 0 & 0 & 17 & 0.34 & 17 & 0.3 & - & - \\
\hline Se. sintoni & 33 & 4.27 & 791 & 15.82 & 824 & 14.28 & 106.25 & 239.48 \\
\hline Total & 773 & 13.39 & 4999 & 86.61 & 5772 & 100 & - & - \\
\hline
\end{tabular}

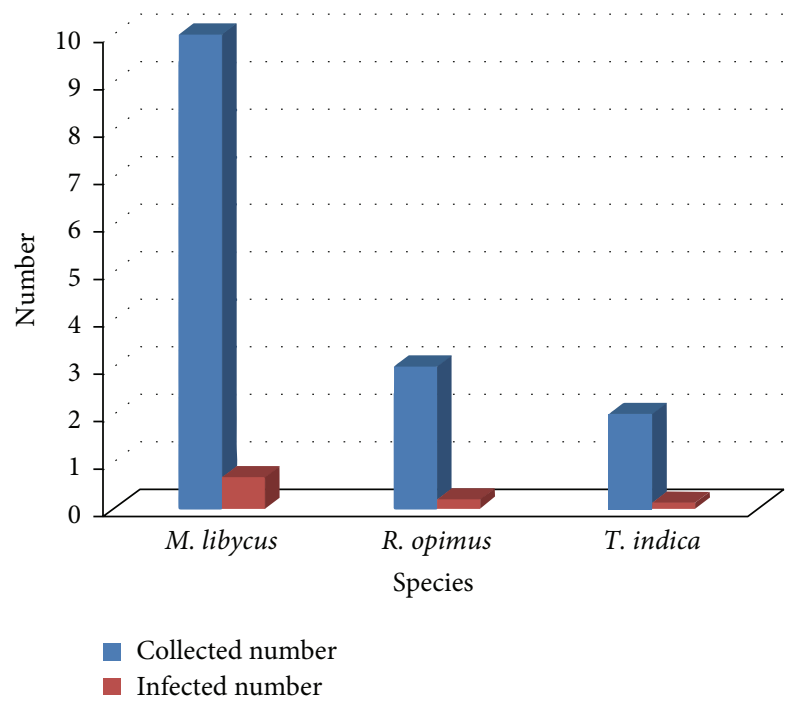

Figure 1: Trapped rodents and their infection to Leishmania parasite, Khatam County, Yazd Province, central Iran, 2006.

The monthly activity of two main species, that is, Ph. papatasi and Se. sintoni both indoors and outdoors, was studied. Both species were collected in all sampling months, from May to October with 2 peaks of activity (Figures 2 and 3).

Sex ratio for $P$ h. papatasi was calculated as 383.55 indoors and 159.76 outdoors. This ratio for Se. sintoni was found to be 106.25 and 239.48 in indoor and outdoor resting places, respectively.

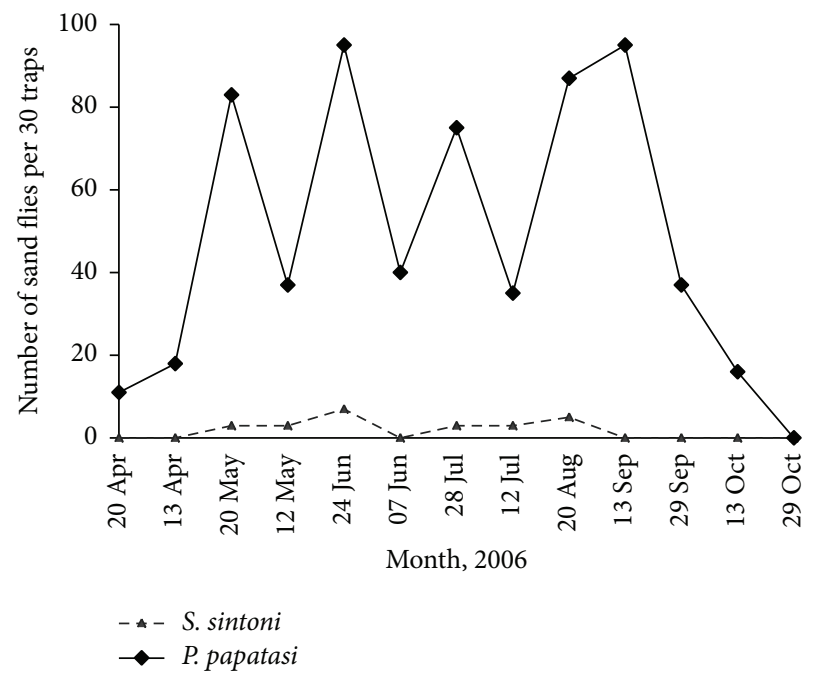

FIGURe 2: Monthly prevalence of Se. sintoni, Ph. papatasi indoors of Khatam County, Yazd Province, central Iran, 2006.

During August and September a total of 295 female sand flies were dissected for the parasitic infection, but finding the promastigote was failed.

Susceptibility test with Deltamethrin $0.025 \%$ resulted in $100 \%$ mortality in tested Ph. papatasi.

\section{Discussion}

This is the first time that an epidemiological study was conducted in Khatam County. In this survey six sand fly species 


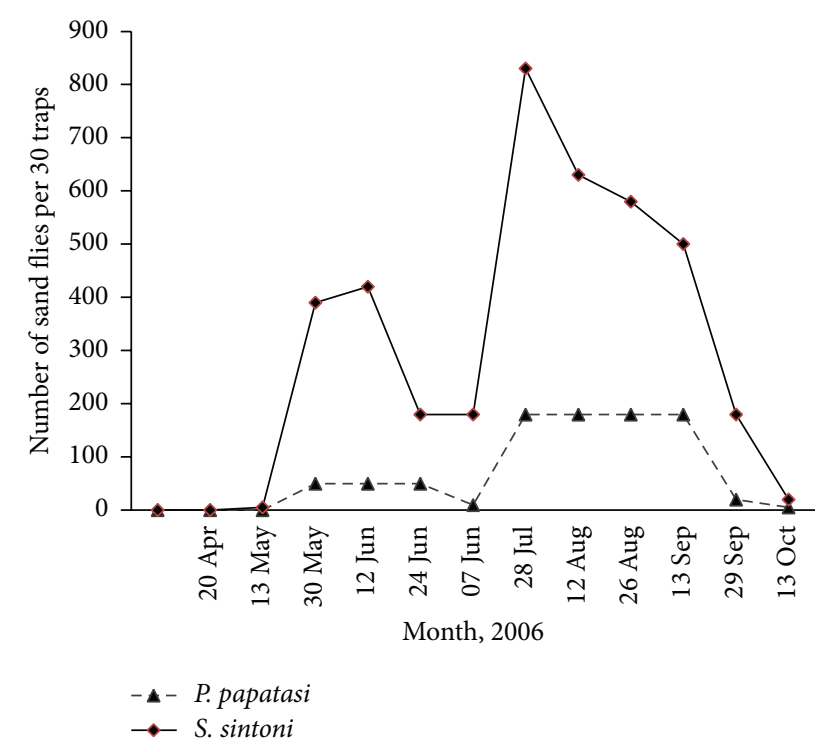

FIGURE 3: Monthly activity of Se. sintoni, Ph. papatasi outdoors of Khatam County, Yazd Province, central Iran, 2006.

were collected and identified; among them $P h$. papatasi was the dominant species indoors (95.2\%). This high indoor density confirms the endophilicity behavior of this sand fly and proves its domestic comparing other collected species. It is also found that because of neighborhood of rodent burrows and villages Se. sintoni had a relative high density indoors. This is the same as some other parts of Iran $[7,18]$.

Although the promastigotes of Leishmania parasite were not found in dissected $\mathrm{Ph}$. papatasi specimens, high density of this species in both indoor and outdoor resting places, as well as isolation of L. major from this sand fly in close foci, that is, Ardakan, seems to be the main vectors of cutaneous leishmaniasis in the study area and has a critical role in the disease transmission from rodents to human $[19,20]$. Test of Ph. papatasi against Deltamethrin 0.05 showed it is susceptible. Although there is a report of tolerance to DDT $4 \%$ in this species [21], it is now susceptible to all tested insecticides. Therefore, these insecticides can be used in epidemics of zoonotic cutaneous leishmaniasis for sand fly control [22, 23].

With due attention to leptomonad infection of all three captured rodents, detection of $L$. major from R. opimus, and history of studies in Iran, R. opimus and M. libycus are introduced as reservoir hosts of ZCL in Khatam County. These gerbils have been found to be infected in central and northeastern foci of the disease in Iran $[2,3,6,7,9,20,21,24-$ 27].

Study on prevalence of the disease in the inhabitant population of Khatam County showed the acute lesions existed in all age groups and both genders $(\alpha=0.05)$. This means we encounter a new focus of cutaneous leishmaniasis. As L. major was isolated from native people without history of travel, it can be concluded that ZCL has prevalence in the county. This parasite is the causative agent of ZCL in other foci of the disease in Iran [7]. So, new focus of ZCL has been established in Khatam County with L. major as agent, $P h$. papatasi as suspected vector, and R. opimus as the main reservoir host. It also seems that $M$. libycus and T. indica play a role in preserving this focus with $R$. opimus.

It is conjectured that development of agriculture in the area, especially cultivating crops like sugar beet and vegetables, and construction of numerous water wells for the agricultural purposes have increased the soil moisture and resulted in the colonization of gerbils and increasing the population of sand flies.

Outdoor sleeping behavior of people during the disease transmission months in summer and close contact of farmers with the infected sand flies in farms have resulted in increasing the rate of human infection.

For control of the disease it is suggested to start a rodent control program within a radius of $500 \mathrm{~m}$ around the infected/at risk villages prior to starting the active season of sand flies. Health education and improving the knowledge of people regarding ZCL can help to prevent the disease transmission. Self-protection using long-lasting insecticide treated net as well as repellents can also decrease the humanvector contact and therefore collapse the disease prevalence in the area.

\section{Conclusion}

In this study, $P$. papatasi and S. sintoni were, respectively, predominant species both indoors and outdoors. T. indica, $M$. libycus, and R. opimus were found to be infected with amastigotes of Leishmania parasite. Study of prevalence among 1364 inhabitants of Marvast and Harat district showed rate of $4.95 \%$ for active lesions and the scar rate was $9.75 \%$ but our studies showed that the majority of scars were due to the treatment of ulcers in the same year. Based on the findings of this study, zoonotic cycle of the disease exists in the area. Therefore planning for the disease control should be based on this form.

\section{Conflict of Interests}

The authors declare that there is no conflict of interests regarding the publication of this paper.

\section{Acknowledgments}

Authors wish to thank and appreciate Dr. Salari honorable Director of Yazd Health Research Station, Dr. Nouri Shadkam honorable Health Deputy of Yazd Province, Dr. Jafari Health Network Director of Khatam Township, and Dr. Forghani and Mr. Ezzat, experts of Yazd Province Health Center, for their sincere cooperation for better accomplishment of this project. They thank Mr. Kafi, Mr. Noroozi, and Mr. Mousavi who are experts of health network for their cooperation in field operations. This research has been done with support of scientific pole of Health Research Institute, Tehran University of Medical Sciences (Project no. t-241/68/). 


\section{References}

[1] WHO, "Report of the consultative meeting on cutaneous leishmaniasis," March 2010, http://www.who.int/leishmaniasis/ resources/Cutaneous_leish_cm_2008.pdf.

[2] R. Jafari, Study of cutaneous leishmaniasis in Nikabad town [M.S. thesis of Health Sciences in Medical Insectology and Fighting with Vectors Course], Tehran University of Medical Sciences, Tehran, Iran, 1997.

[3] E. Javadian, A. Nadim, G. H. Tahvildare-Bidruni, and V. Assefi, "Epidemiology of cutaneous leishmaniasis in Iran: B. Khorassan. Part V: report on a focus of zoonotic cutaneous leishmaniasis in Esferayen," Bulletin de la Societe de Pathologie Exotique et de ses Filiales, vol. 69, no. 2, p. 140, 1976.

[4] H. Kasiri, E. Javadian, and M. A. Seyedi-Rashti, "Liste des phlebotominae (Diptera: Psychodidae) d'Iran," Bulletin de la Société de Pathologie Exotique, vol. 93, no. 2, pp. 129-130, 2000.

[5] R. P. Lane and R. W. Crosskey, Medical Insects and Arachnids, Springer, 1993.

[6] M. R. Yaghoobi-Ershadi, A. A. Akhavan, and M. Mohebali, "Meriones libycus and Rhombomys opimus (Rodentia: Gerbillidae) are the main reservoir hosts in a new focus of zoonotic cutaneous leishmaniasis in Iran," Transactions of the Royal Society of Tropical Medicine and Hygiene, vol. 90, no. 5, pp. 503504, 1996.

[7] M. R. Yaghoobi-Ershadi, A. A. Akhavan, A. V. ZahraeiRamazani et al., "Epidemiological study in a new focus of cutaneous leishmaniasis in the Islamic Republic of Iran," Eastern Mediterranean Health Journal, vol. 9, no. 4, pp. 816-826, 2003.

[8] M. R. Yaghoobi-Ershadi, A. A. Hanafi-Bojd, A. A. Akhavan, R. Jafari, and M. Mohebali, "Study of animal sources of cutaneous leishmaniasis in two epidemic focuses in Yazd province," Scientific Research Magazine of Yazd University of Medical Sciences, vol. 2, pp. 38-43, 2001.

[9] M. R. Yaghoobi-Ershadi, A. A. Hanafi-Bojd, A. A. Akhavan, A. R. Zahrai-Ramazani, and M. Mohebali, "Epidemiological study in a new focus of cutaneous leishmaniosis due to Leishmania major in Ardestan town, central Iran," Acta Tropica, vol. 79, no. 2, pp. 115-121, 2001.

[10] M. R. Yaghoobi-Ershadi and E. Javadian, "Epidemiological study of reservoir hosts in an endemic area of zoonotic cutaneous leishmaniasis in Iran," Bulletin of the World Health Organization, vol. 74, no. 6, pp. 587-590, 1996.

[11] M.-R. Yaghoobi-Ershadi, A.-R. Zahraei-Ramazani, A.-A. Akhavan, A.-R. Jalali-Zand, H. Abdoli, and A. Nadim, "Rodent control operations against zoonotic cutaneous leishmaniasis in rural Iran," Annals of Saudi Medicine, vol. 25, no. 4, pp. 309-312, 2005.

[12] A. Karimi, A. A. Hanafi-Bojd, M. R. Yaghoobi-Ershadi, A. A. Akhavan, and Z. Ghezelbash, "Spatial and temporal distributions of phlebotomine sand flies (Diptera: Psychodidae), vectors of leishmaniasis, in Iran," Acta Tropica, vol. 132, no. 1, pp. 131-139, 2014.

[13] M. Yaghoobi-Ershadi, "Phlebotomine sand flies (Diptera: Psychodidae) in Iran and their role on leishmania transmission," Journal of Arthropod-Borne Diseases, vol. 6, no. 1, pp. 1-17, 2012.

[14] S. Ardehali, H. V. Rezaie, and A. Nadim, Leishmaniasis Parasite an Leishmaniasis, Publication Center of Tehran University, Tehran, Iran, 2nd edition, 1998.

[15] A. Etemad, "Rodents and their detection key," in Iran Mammals, vol. 1, pp. 288-298, National Institute of Natural Resources and Human Environment Preservation, Tehran, Iran, 1978.
[16] G. H. Edrissian, Z. Zovein, and A. Nadim, "A simple technique for preparation of smears from the ear of Rhombomys opimus for the detection of leishmanial infection," Transactions of the Royal Society of Tropical Medicine and Hygiene, vol. 76, no. 5, pp. 706-707, 1982.

[17] O. Theodor, Revue de la Faculté des Sciences de l'Université d'Istanbul Série B, vol. 17, p. 107, 1952.

[18] A. A. Akhavan, M. R. Yaghoobi-Ershadi, and M. Mohebali, "Epidemiology of dermal leishmaniasis (human infection) in the region of badrood, natanz township," in Proceedings of the 1st Congress and Reeducation of Iran Medical Insectology, Tehran, Iran, 2005.

[19] M. A. Seyedi-Rashti and A. Nadim, "Cutaneous leishmaniasis in Baluchistan, Iran," in Proceedings of the 11th International Congress for Tropical Medicine and Malaria, Abstract and Poster, Calgary, Canada, September 1984.

[20] M. A. Seyedi-Rashti and A. Salehzadeh, "A new focus of zoonotic cutaneous leishmaniasis near Tehran, Iran," Bulletin de la Société Française de Parasitologie, vol. 8, 1990.

[21] M. R. Yaghoob-Ershadi and E. Javadian, "Susceptibility of Phlebotomus papatasi to DDT in the most important focus of zoonotic cutaneous leishmaniasis, Isfahan province, Iran," Journal of Entomological Society of Iran, vol. 12, no. 13, pp. 27-37, 1993.

[22] A. A. Afshar, Y. Rassi, I. Sharifi et al., "Susceptibility status of Phlebotomus papatasi and P. sergenti (Diptera: Psychodidae) to DDT and deltamethrin in a focus of cutaneous leishmaniasis after earthquake strike in Bam, Iran," Iranian Journal of Arthropod-Borne Diseases, vol. 5, no. 2, pp. 32-14, 2011.

[23] M. A. Seyedi-Rashti, H. Yazdan-panah, H. Shah-Mohammadi, and M. Jedari, "Susceptibilityof Phlebotomus papatasi (Diptera: Psychodidae) to D.D.T. in some foci of cutaneous leishmaniasis in Iran," Journal of the American Mosquito Control Association, vol. 8, no. 1, pp. 99-100, 1992.

[24] E. Javadian, "Reservoir host of cutaneous leishmaniasis in Iran," in Proceedings of the 12th International Congress for Tropical Medicine and Malaria, Amsterdam, The Netherlands, September 1988.

[25] E. Javadian, M. Dehestani, N. Nadim et al., "Confirmation of Tatera indica (Rodentia: Gerbilldae) as the main reservoir host of zoonotic cutaneous leishmaniasis in the west of Iran," Iranian Journal of Public Health, vol. 27, no. 1-2, 1998.

[26] A. Nadim, M. A. Seyedi-Rashti, and A. Mesghali, "Epidemiology of cutaneous leishmaniasis in Turkemen Sahara, Iran," The Journal of Tropical Medicine and Hygiene, vol. 71, no. 9, pp. 238239, 1968.

[27] M. R. Yaghoobi-Ershadi, R. Jafari, and A. A. Hanafi-Bojd, "A new epidemic focus of zoonotic cutaneous leishmaniasis in central Iran," Annals of Saudi Medicine, vol. 24, no. 2, pp. 98$101,2004$. 


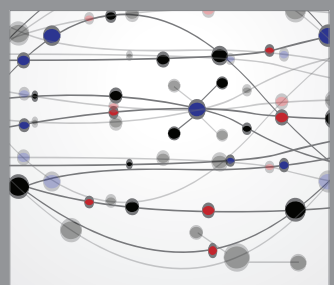

The Scientific World Journal
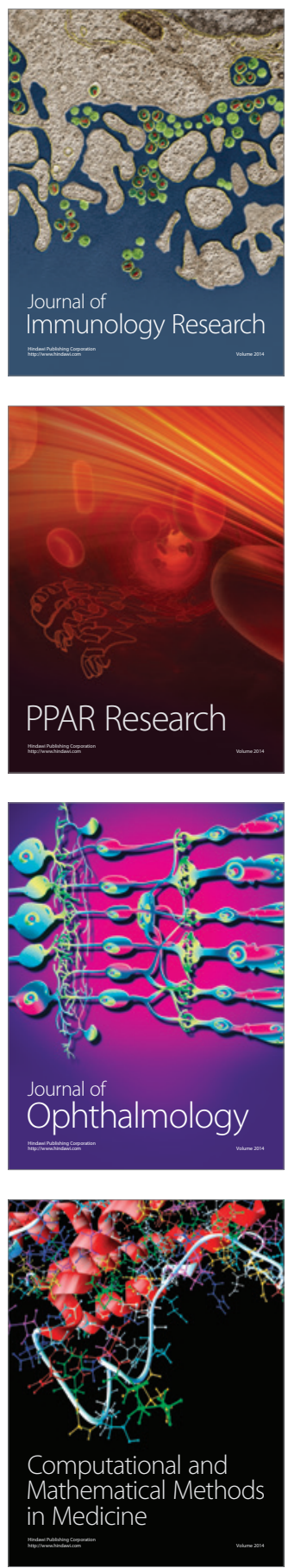

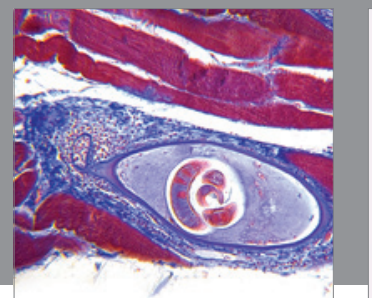

Gastroenterology

Research and Practice
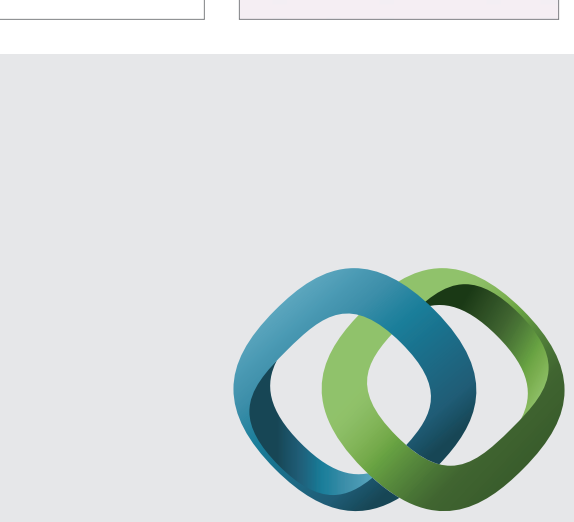

\section{Hindawi}

Submit your manuscripts at

http://www.hindawi.com
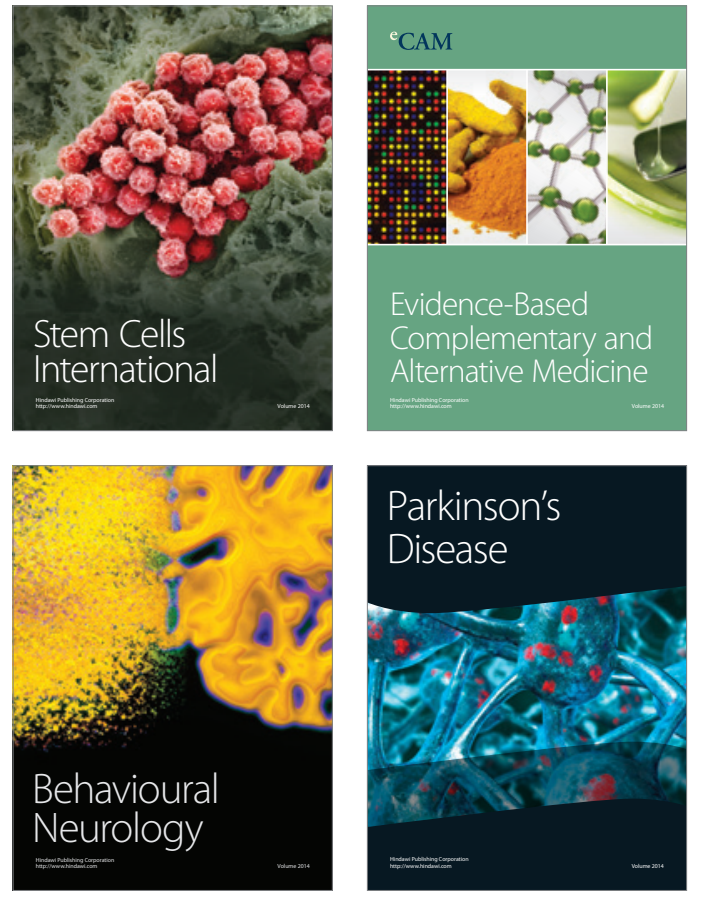
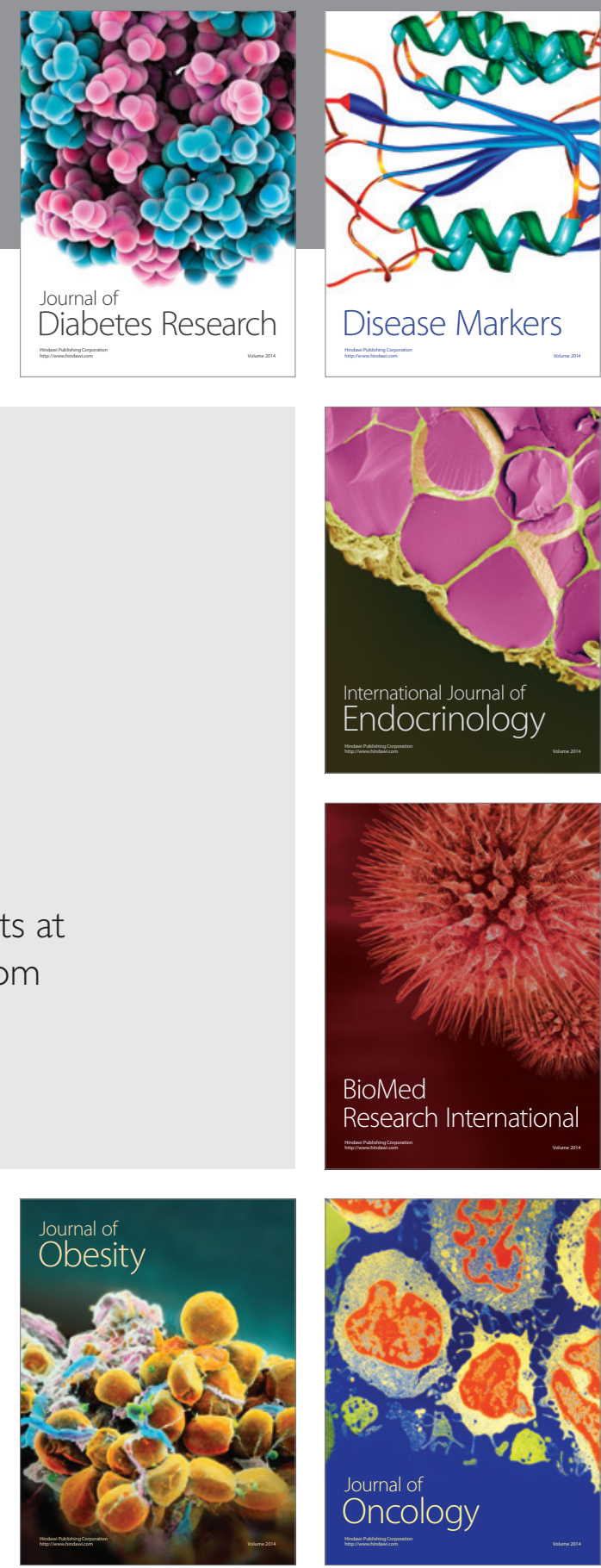

Disease Markers
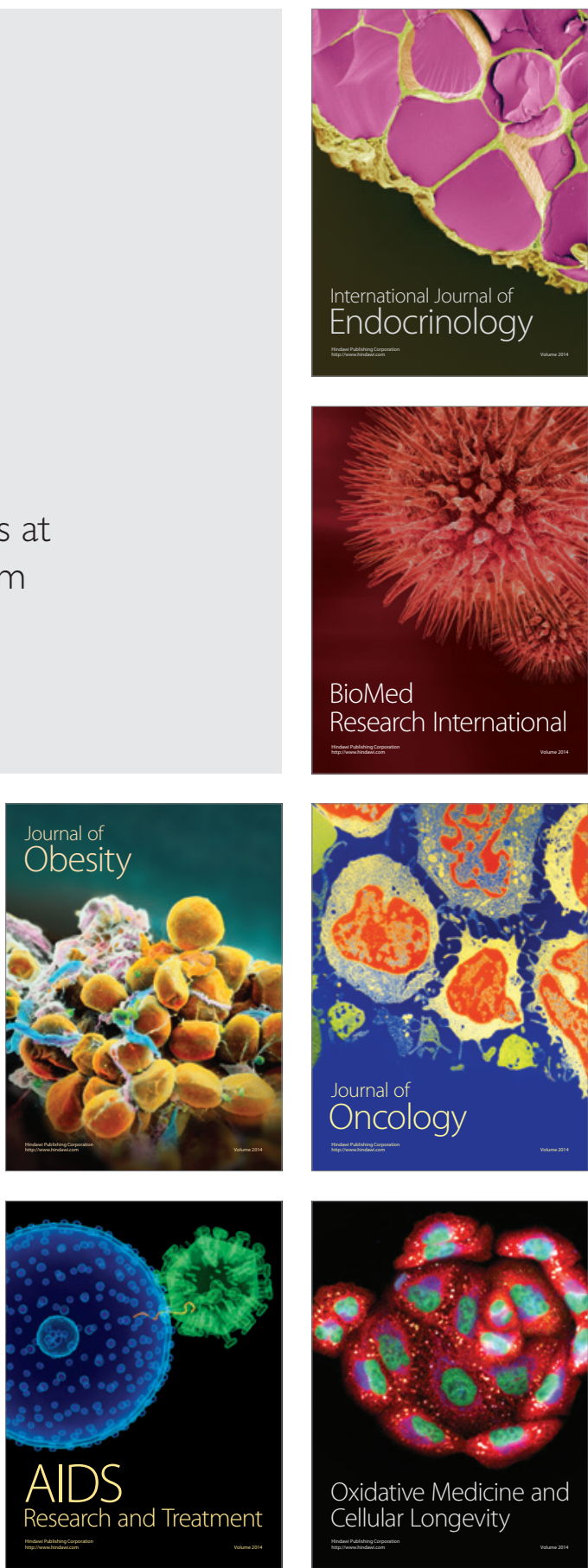